\title{
Exercise-Induced Rhabdomyolysis: A Case Report and Literature Review
}

\author{
Amira Al Badi ${ }^{1}$, Sara Al Rasbi ${ }^{1}$, Abdullah M. Alalawi ${ }^{1}$ \\ 1. Medicine, Sultan Qaboos University Hospital, Muscat, OMN
}

Corresponding author: Abdullah M. Alalawi,dr.abdullahalalawi@gmail.com

\begin{abstract}
A 19-year-old man presented to the ED with bilateral leg pain and dark discoloration of the urine after he started an intense aerobic exercise. Blood workup showed significantly elevated creatine kinase (CK), acute kidney injury (AKI), and disseminated intravascular coagulation (DIC). The patient had a double-incision, bilateral fasciotomy with debridement to relieve the bilateral, lower-limb, compartment syndrome following admission. Also, his kidney function deteriorated, requiring several sessions of hemodialysis. His hospital stay was complicated by multidrug-resistant (MDR) Acinetobacter baumannii bacteremia. After three weeks of hospital admission, the patient was discharged home with a follow-up outpatient physiotherapy for bilateral foot drop, which showed a remarkable recovery eventually. This case highlights the potentially lifethreatening risks associated with unaccustomed physical exercise and emphasizing the essential preventive measures to reduce the risk of developing exercise-induced rhabdomyolysis. We present the pathophysiology of exercise-induced rhabdomyolysis, clinical presentation, diagnosis, treatment, and prognosis.
\end{abstract}

Categories: Emergency Medicine, Internal Medicine, Nephrology

Keywords: rhabdomyolysis, exercise, compartment syndrome, creatine kinase, case report

\section{Introduction}

Exertional or exercise-induced rhabdomyolysis is a condition caused by unaccustomed physical exercise and characterized by a breakdown of skeletal muscles that leads to the release of its intracellular components, such as myoglobin and creatine kinase (CK), into the circulatory system [1-2]. It can cause severe complications, including acute kidney injury (AKI), disseminated intravascular coagulation (DIC), compartment syndrome, cardiac arrhythmia, liver dysfunction, and various electrolyte derangements such as hypocalcemia, hypercalcemia, hyperkalemia, hyperphosphatemia, hypomagnesemia, and hyperuricemia, along with mortality risk [3-6]. Exercise-induced rhabdomyolysis occurs in the setting of intense physical exercise, prolonged physical activity or sudden and excessive muscle contractions, and symptoms include change in the color of the urine, muscle ache and pain, headache, and fatigue [1]. Rhabdomyolysis has been increasingly diagnosed worldwide due to the increased popularity of physical activity and exercise [2, 5, 7]. We report a case of a young man who started an intense aerobic exercise before military recruitment,

Received 08/11/2020

Review began 08/13/2020 Review ended 08/18/2020 Published 08/26/2020

\section{Copyright 2020}

Al Badi et al. This is an open access article distributed under the terms of the Creative Commons Attribution License CC-BY 4.0., which permits unrestricted use, distribution, and reproduction in any medium, provided the original author and source are credited. and had exercise-induced rhabdomyolysis, causing severe organ dysfunctions. This report presents the pathophysiology of exercise-induced rhabdomyolysis, clinical presentation, diagnosis, treatment, and prognosis.

\section{Case Presentation}

A 19-year-old man, previously healthy and not on any regular medications, presented to the ED at Sultan Qaboos University Hospital (SQUH) in July 2019 with a one-day history of generalized fatigue, bilateral leg pain, and dark discoloration of the urine. There was no history of fever, chills, nausea, vomiting, or diarrhea. Moreover, there was no history of consuming alcohol, anabolic steroid, or drug abuse. His symptoms started a few hours after jogging for around $26 \mathrm{~km}$ to improve his fitness before military recruitment. However, he was barely practicing exercise before this attempt.

On presentation to the ED, he was alert and oriented but appeared to be in pain. He was clinically dehydrated, and his vitals were as follows: temperature $37.0^{\circ} \mathrm{C}$, blood pressure $130 / 70 \mathrm{mmHg}$, heart rate 100 bpm and regular, and oxygen saturation $100 \%$ on room air. He had generalized bilateral lower limb swelling and tenderness, but the neurovascular examination was intact. Chest, cardiovascular, and abdominal examinations were unremarkable.

Laboratory findings are presented in Table 1. As summarized, the patient presented with high CK, AKI (using RIFLE criteria), DIC, and deranged liver enzymes. His initial venous blood gas showed uncompensated high anion gap metabolic acidosis, which could be explained by severe dehydration, AKI, and exhaustion. His initial electrocardiogram (ECG) and chest X-ray were normal. Immediately, using a combination of normal saline and sodium bicarbonate, IV fluid resuscitation was initiated, targeting urine output between 200 and $300 \mathrm{ml} / \mathrm{h}$. Within the first day of admission, he experienced worsening of the bilateral, lower limb swelling and increased pain requiring opioids. Because of the high probability of acute compartment syndrome, the 


\section{Cureus}

patient underwent double-incision, bilateral fasciotomy with debridement to relieve the bilateral, lower limb, compartment syndrome.

\begin{tabular}{|c|c|c|}
\hline Test & Normal range & Admission day \\
\hline Hemoglobin (g/dL) & 11.5-15.5 & 18.5 \\
\hline Hematocrit (L/L) & $0.350-0.450$ & 0.5 \\
\hline WBC $\left(10^{9} / \mathrm{L}\right)$ & $2.2-10.0$ & 33.2 \\
\hline Neutrophil count $\left(10^{9} / \mathrm{L}\right)$ & $1.0-5.0$ & 30.2 \\
\hline Venous pH & 7.35-7.45 & 7.13 \\
\hline $\mathrm{HCO} 3$ (mmol/L) & 21.8-26.9 & 14.7 \\
\hline pCO2 (mmHg) & $32.0-45.0$ & 44.4 \\
\hline Anion gap & $5.0-13.0$ & 17.3 \\
\hline Lactate $(\mathrm{mmol} / \mathrm{L})$ & $0.5-1.6$ & 6.5 \\
\hline CK (U/L) & $39-308$ & 587,600 \\
\hline Creatinine $(\mu \mathrm{mol} / \mathrm{L})$ & $59-104$ & 167 \\
\hline Potassium (mmol/L) & $3.5-5.1$ & 4.2 \\
\hline Sodium (mmol/L) & 135-145 & 136 \\
\hline Alanine transaminase $(\mathrm{U} / \mathrm{L})$ & $0-41$ & 2561 \\
\hline Aspartate aminotransferase (U/L) & $0-40$ & 6632 \\
\hline Alkaline phosphatase $(\mathrm{mmol} / \mathrm{L})$ & $40-129$ & 140 \\
\hline Total bilirubin $(\mu \mathrm{mol} / \mathrm{L})$ & $0-17$ & 19 \\
\hline INR & 0.91-1.09 & 2.65 \\
\hline aPTT (second) & 26.4-38.1 & 53 \\
\hline TT (second) & 12.8-17.6 & 24.4 \\
\hline Fibrinogen $(g / L)$ & $1.7-3.6$ & 1.4 \\
\hline Calcium (mmol/L) & 2.15-2.55 & 1.74 \\
\hline Uric acid (mmol/L) & $0.20-0.45$ & 0.68 \\
\hline Phosphate (mmol/L) & $0.81-1.45$ & 2.05 \\
\hline \multicolumn{3}{|l|}{ Urine Dipstick: } \\
\hline Urine glucose & & Negative \\
\hline Urine bilirubin & & Negative \\
\hline Urine blood & & Positive (3+) \\
\hline Urine $\mathrm{pH}$ & & 6 \\
\hline Urine protein & & Trace \\
\hline
\end{tabular}

TABLE 1: Blood and urine tests results on the day of admission.

aPTT, activated partial thromboplastin time; TT, thrombin time; CK, creatine kinase; WBC, white blood cell; INR, international normalized ratio

Despite adequate rehydration, renal function continued to deteriorate, evidenced by 


\section{Cureus}

worsening creatinine levels (Figure 1), persistent hyperkalemia (Figure 2), and oliguria ( Figure 3). The patient underwent the first dialysis session within two days after admission. On the ninth day, the patient became unwell, febrile, and tachycardia. After a sepsis workup, the patient was started empirically on meropenem and vancomycin. The blood cultures sampled from the dialysis line and peripheral vein grew for multidrug-resistant (MDR) Acinetobacter baumannii. Besides replacing the dialysis line, the patient received a 10-day course of meropenem and a high dose of tigecycline as recommended by the infectious disease team. Overall, the patient showed a clinical and biochemical response to the administered antibiotics.

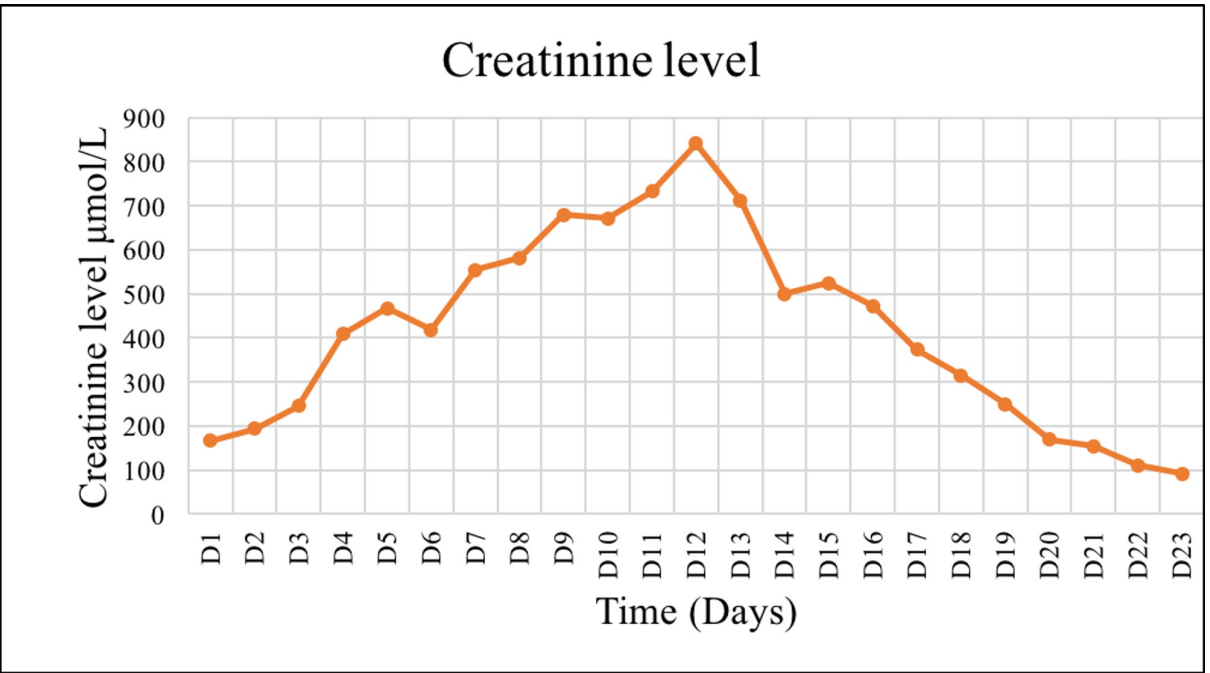

FIGURE 1: Trend of creatinine level reflecting kidney function during admission.

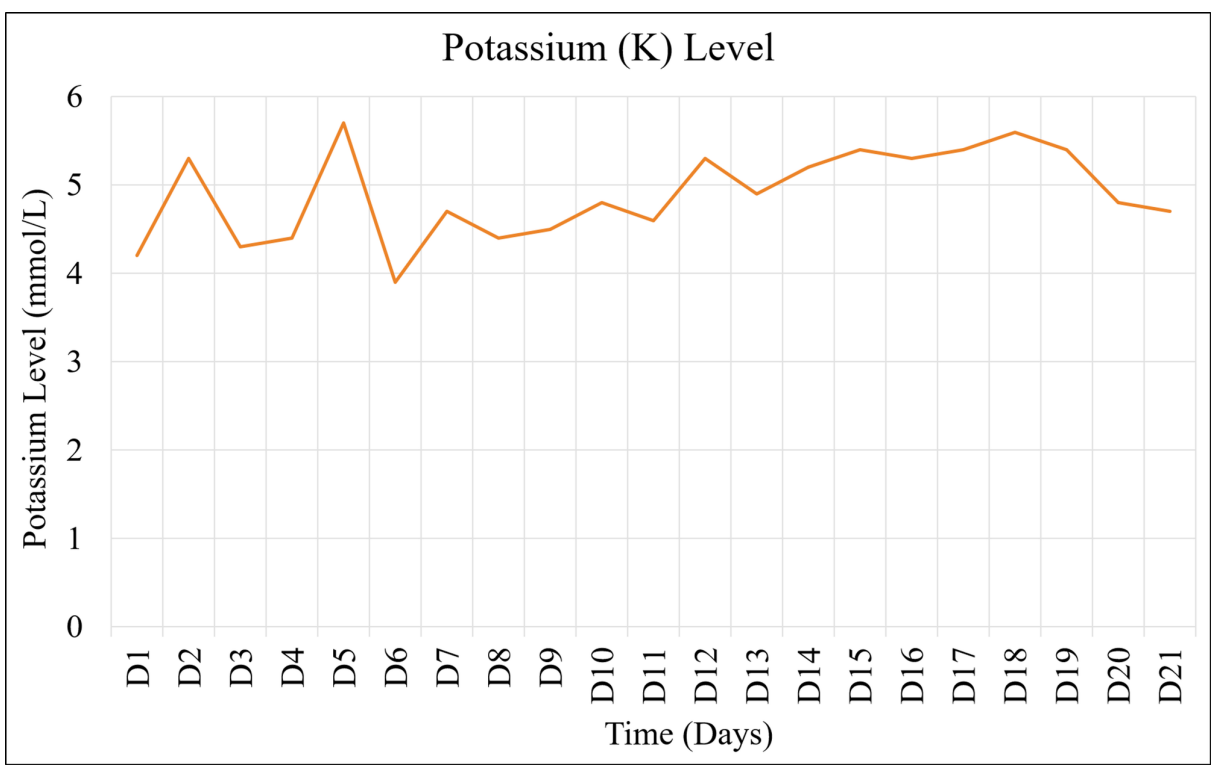

FIGURE 2: The trend of potassium (K) level during admission. 


\section{Cureus}

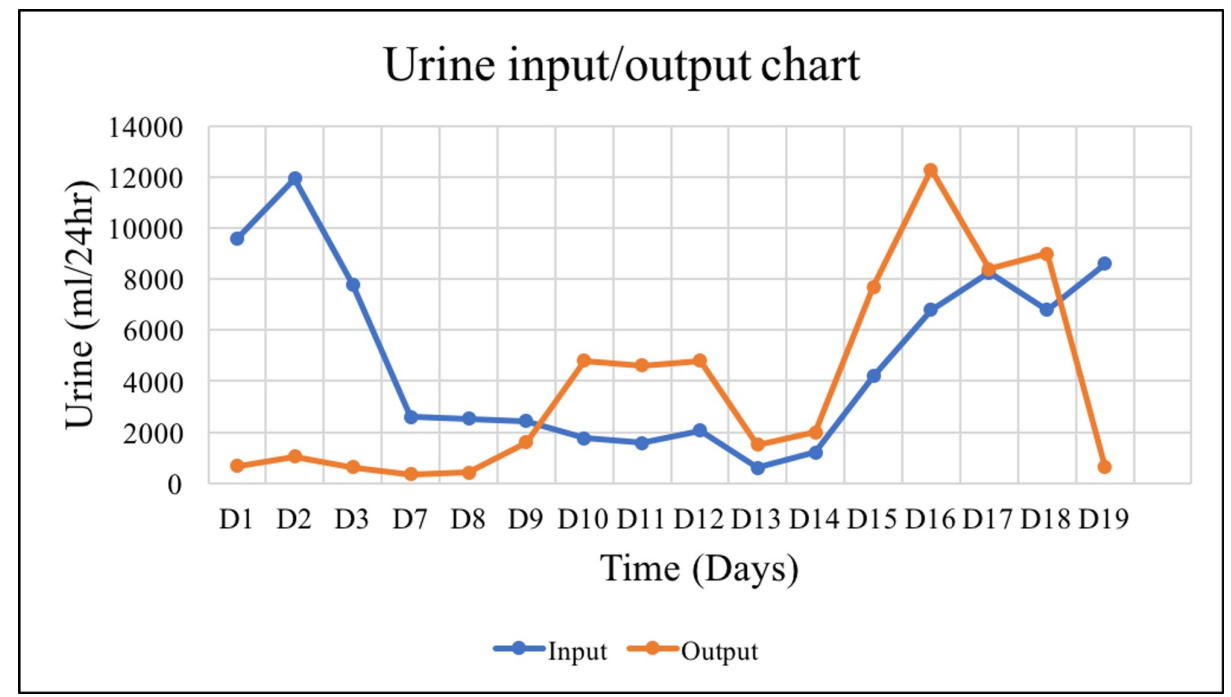

FIGURE 3: Fluid intake/urine output chart.

In summary, the patient completed four sessions of tissue debridement, followed by the closure of fasciotomy wounds and six sessions of hemodialysis. The patient's kidney function (Figures 1-3), coagulopathy measured by INR (Figure 4), and CK (Figure 5) gradually improved. He was discharged as soon as kidney function returned to normal. He also had bilateral foot drop, which improved remarkably with physiotherapy. Otherwise, he remained well upon follow up.

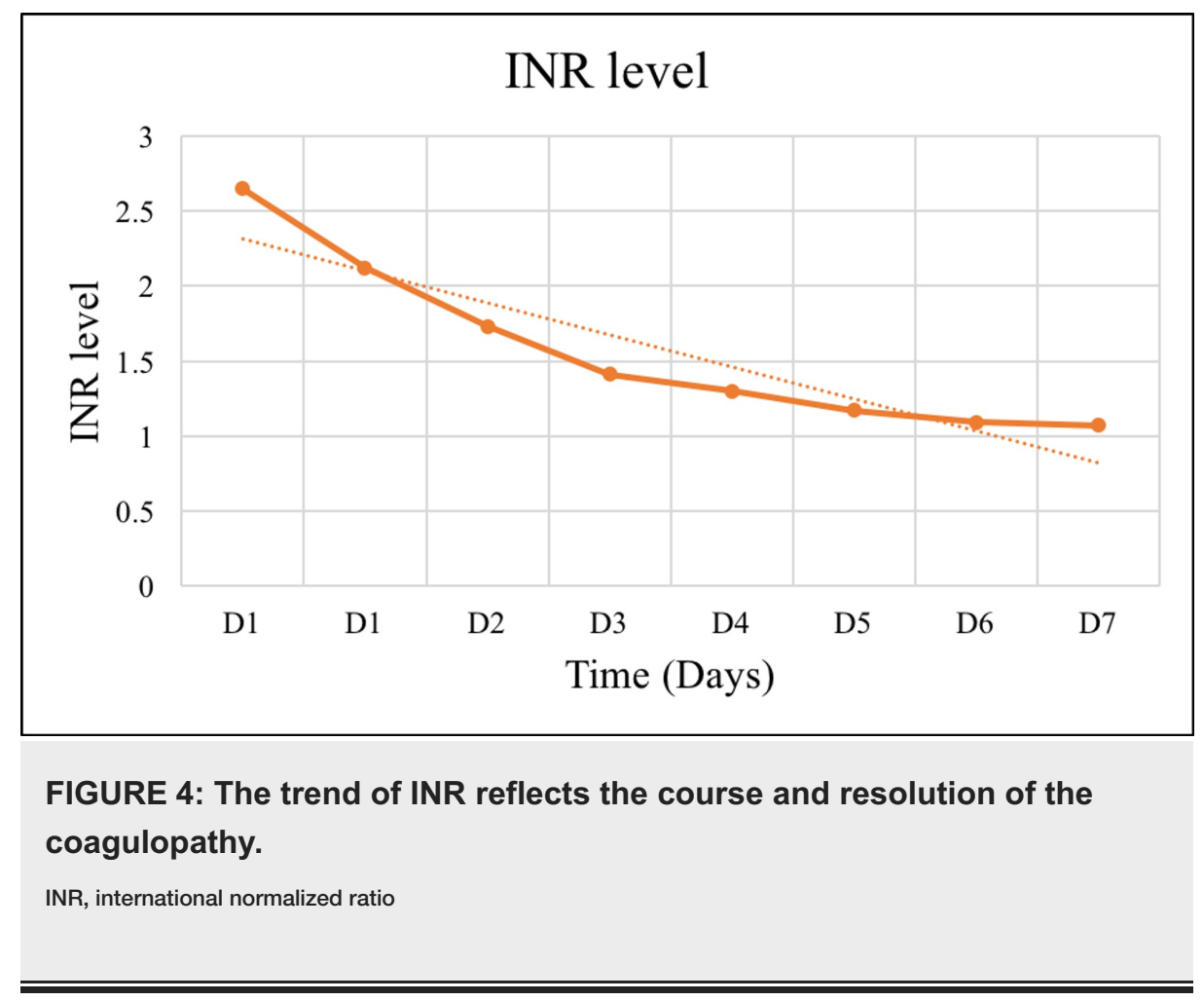




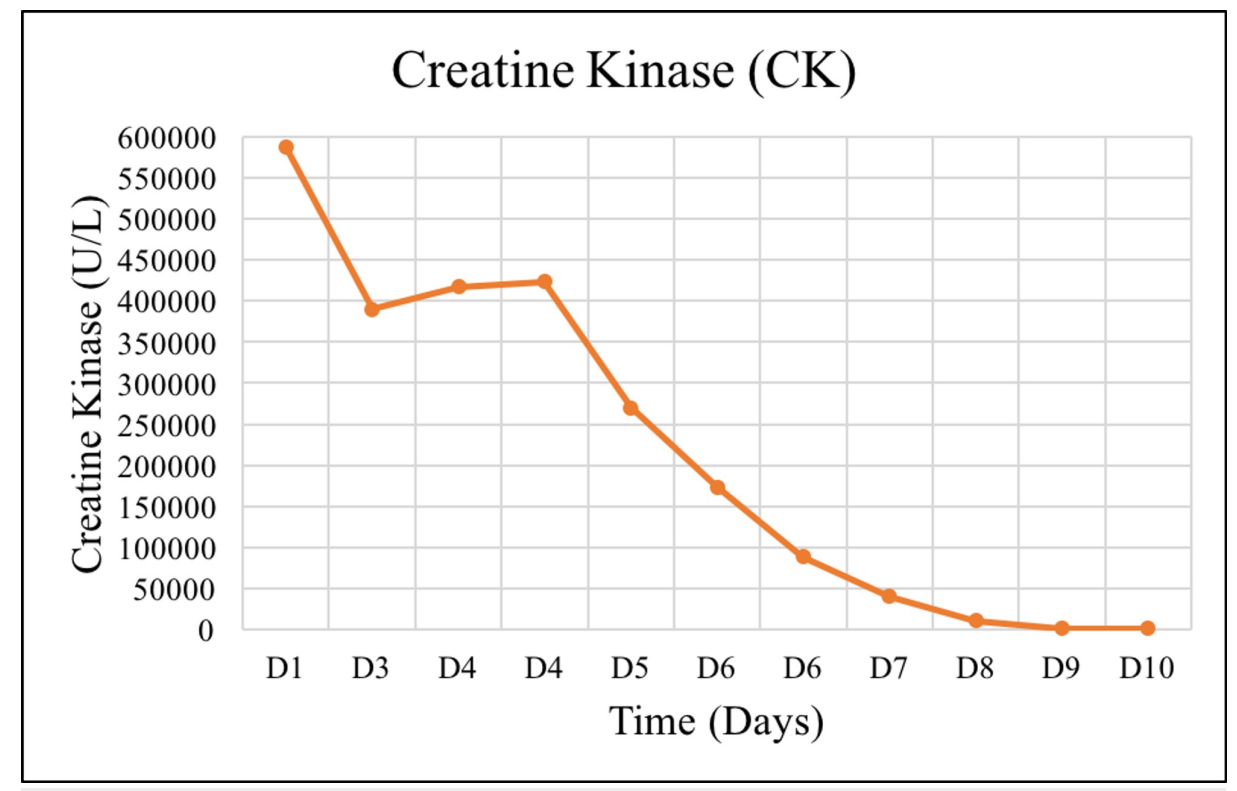

FIGURE 5: The trend of CK measured during admission.

CK, creatine kinase

\section{Discussion}

Primary factors that cause exercise-induced rhabdomyolysis include poor body fitness combined with prolonged-duration, high-intensity, and weight-bearing exercise (e.g., eccentric contraction, downhill running) [1, 8]. Other predisposing factors include dehydration, hot environment [3], genetic mutations (e.g., Caveolin-3) [4, 9], underlying myopathy [10, 11], drugs such as terbinafine and statin [12], obesity, and tobacco use [13]. Furthermore, sickle cell trait has shown to increase the risk of exercise-induced rhabdomyolysis, even with minor trauma [13].

Intense exercise depletes the adenosine triphosphate (ATP) required by the pumps and channels that regulate calcium $(\mathrm{Ca} 2+)$ in the sarcoplasmic reticulum, which results in higher resting levels of $\mathrm{Ca} 2+$ in muscle cells [1-2]. The increased levels of $\mathrm{Ca} 2+$ boost the activity of proteases and phospholipase A2, causing damage to desmin, dystrophin, and other cytoskeleton support structures [1]. The cytoskeleton collapse facilitates muscle membrane tears followed by an increase in the cell membrane permeability, excessive release of intracellular proteins, including CK and myoglobin, an increase in Ca2+ influx, and additional cellular damage $[10,14]$. This necrotic process triggers a robust inflammatory response and a subsequent regeneration process $[2,15]$. Muscle cellular membrane dysfunction and inflammatory reactions cause an accumulation of intracellular fluids and an increase in muscle compartment pressure, and, thus, worse muscle cell ischemia and local edema eventually lead to acute compartment syndrome [16].

The incidence of AKI in patients with exercise-induced rhabdomyolysis is estimated to be between $10 \%$ and $30 \%[1,17]$. The mortality risk can be as high as $59 \%$, even in an ICU setting [18]. The primary causes of AKI are renal vasoconstriction secondary to myoglobin, hypovolemia, elevated circulating endotoxins and cytokines, and enhanced sympathetic tone and renin-angiotensin-aldosterone system activation [1]. Myoglobin effects contributing to kidney damage include the formation of casts in the distal convoluted tubules and the direct toxicity of myoglobin in the proximal convoluted tubules [3].

Muscle pain, weakness, fatigue, and dark discoloration of urine are classical symptoms of exercise-induced rhabdomyolysis [5]. An initial diagnosis is typically made if CK levels are elevated more than five times the upper limit [5], and there is a history of recent strenuous physical activity.

Treatment includes an IV infusion of crystalloid fluids using normal saline with or without Ringer's lactate to achieve urine output between 200 and $300 \mathrm{~mL} / \mathrm{h}$ [5]. If systemic acidosis is present, sodium bicarbonate should also be administered to ensure urine $\mathrm{pH}$ is higher than 6.5 to minimize myoglobin-induced kidney damage [3-4]. However, the evidence that suggests urinary alkalization is more effective than hydration alone in reducing AKI incidence $[17,19]$ is weak. If compartment syndrome is suspected, prompt orthopedic consultation is warranted to evaluate intra-compartmental pressure, and, if necessary, perform a fasciotomy to avoid or minimize damage caused by ischemic necrosis of skeletal muscles [3, 20].

Our patient had the full spectrum of exercise-induced rhabdomyolysis associated complications, 
accompanied by a hospital-acquired and potentially deadly infection. The patient's exercise routine was unusual, where he jogged more than $26 \mathrm{~km}$ per day in hot weather. His body temperature was $37.0^{\circ} \mathrm{C}$, which is not consistent with a heatstroke diagnosis. Blood tests also indicated AKI, DIC, and several electrolytes derangements along with deranged liver enzymes. The patient also developed bilateral, lower-limb, compartment syndrome that required a bilateral double fasciotomy and several sessions of wound debridement. Although the adequate and prompt intervention was established, the patient had bilateral foot drop, due to myonecrosis that involved anterior tibial muscles, which improved remarkably with physiotherapy. Despite adequate IV hydration and urinary alkalization, hemodialysis was necessary. His kidney function returned to normal within three weeks after admission. Moreover, during admission, the patient acquired MDR A. baumannii line related bacteremia. He was successfully treated with 10 days of IV antibiotics.

\section{Conclusions}

Although the prevalence of exercise-induced rhabdomyolysis has increased, this case has shown potentially added complications such as healthcare-associated infection, which might be life-threatening. Measures such as warming-up and periodic repetition of eccentric exercises, along with sufficient water intake, should be considered for people who exercise for the first time or those with poor body fitness to aim to prevent exertional rhabdomyolysis.

\section{Additional Information \\ Disclosures}

Human subjects: Consent was obtained by all participants in this study. Conflicts of interest: In compliance with the ICMJE uniform disclosure form, all authors declare the following: Payment/services info: All authors have declared that no financial support was received from any organization for the submitted work. Financial relationships: All authors have declared that they have no financial relationships at present or within the previous three years with any organizations that might have an interest in the submitted work. Other relationships: All authors have declared that there are no other relationships or activities that could appear to have influenced the submitted work.

\section{References}

1. Kim J, Lee J, Kim S, Ryu HY, Cha KS, Sung DJ: Exercise-induced rhabdomyolysis mechanisms and prevention: a literature review. J Sport Health Sci. 2016, 5:324-333. 10.1016/j.jshs.2015.01.012

2. Paulsen G, Benestad HB: Muscle soreness and rhabdomyolysis. Tidsskr Nor Laegeforen. 2019, 139:10.4045/tidsskr.18.0727

3. Chatzizisis YS, Misirli G, Hatzitolios AI, Giannoglou GD: The syndrome of rhabdomyolysis: complications and treatment. Eur J Intern Med. 2008, 19:568-574. 10.1016/j.ejim.2007.06.037

4. Dunphy L, Morhij R, Tucker S, et al.: Rhabdomyolysis-induced compartment syndrome secondary to atorvastatin and strenuous exercise. BMJ Case Rep. 2017, 2017: 10.1136/bcr-2016-218942

5. Chavez L, Leon M, Einav S, Varon J: Beyond muscle destruction: a systematic review of rhabdomyolysis for clinical practice. Crit Care. 2016, 20:10.1186/s13054-016-1314-5

6. Backer HC, Busko M, Krause FG, Exadaktylos AK, Klukowska-Roetzler J, Deml MC: Exertional rhabdomyolysis and causes of elevation of creatine kinase. Phys Sportsmed. 2019, 1-7. 10.1080/00913847.2019.1669410

7. Fardal H, Goransson LG: [Exercise-induced rhabdomyolysis - a new trend?] . Tidsskr Nor Laegeforen. 2016, 136:1537-1541.10.4045/tidsskr.16.0042

8. Lin H, Chie W, Lien H: Epidemiological analysis of factors influencing an episode of exertional rhabdomyolysis in high school students. Am J Sports Med. 2006, 34:481-486. 10.1177/0363546505281243

9. Scalco RS, Gardiner AR, Pitceathly RD, et al.: CAV3 mutations causing exercise intolerance, myalgia and rhabdomyolysis: expanding the phenotypic spectrum of caveolinopathies. Neuromuscul Disord. 2016, 26:504-510. 10.1016/j.nmd.2016.05.006

10. Shukla S, Verma A: Fever, fasting, and rhabdomyolysis in an adult male . Neurol India. 2020, 68:182. 10.4103/0028-3886.279697

11. Minton T, Forrester N, Baba S, Urankar K, Brady S: A rare case of adult-onset LPIN1 associated rhabdomyolysis. Neuromusc Disord. 2020, 30:241-245. 10.1016/j.nmd.2020.01.004

12. Sunder A, Mohanty B: Rhabdomyolysis - exercise induced nightmare . J Assoc Phys India. 2020, 68:77. 10.4103/jfmpc.jfmpc 37018

13. Nelson D, Deuster P, Carter R, Hill O, Wolcott V, Kurina L: Sickle cell trait, rhabdomyolysis, and mortality among U.S. army soldiers. N Engl J Med. 2016, 375:435-442. 10.1056/NEJMoa1516257

14. Yamin C, Meckel Y, Oliveira J, et al.: Genetic aspects of exercise and rhabdomyolysis . Pediatr Endocrinol Rev. 2014, 11:400-408.

15. Zhang MH: Rhabdomyolosis and its pathogenesis. World J Emerg Med. 2012, 3:11-15. 10.5847/wjem.j.issn.1920-8642.2012.01.002

16. Traub BC, Lane MK, Traub JA: Exercise-induced acute bilateral upper-arm compartment syndrome. Case Rep Emerg Med. 2017, 2017:9454782. 10.1155/2017/9454782

17. Huerta-Alardin AL, Varon J, Marik PE: Bench-to-bedside review: rhabdomyolysis -- an overview for clinicians. Crit Care. 2005, 9:158-169. 10.1186/cc2978

18. Stanley M, Adigun R: Rhabdomyolysis. StatPearls Publishing, Treasure Island, FL; 2020.

19. Kolstad K, Solbu MD: Exercise-induced rhabdomyolysis. Tidsskr Nor Laegeforen. 2016, 136:1504. 10.4045/tidsskr.16.0797 


\section{Cureus}

20. Bonasia DE, Rosso F, Cottino U, Rossi R: Exercise-induced leg pain. Asia Pac J Sports Med Arthrosc Rehabil Technol. 2015, 2:73-84. 10.1016/j.asmart.2015.03.003 